\title{
Candidate Causal Variants at the 8p12 Breast Cancer Risk Locus Regulate DUSP4
}

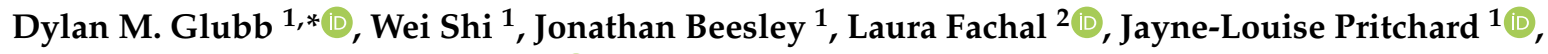 \\ Karen McCue ${ }^{1}$, Daniel R. Barnes ${ }^{3}{ }^{-1}$, Antonis C. Antoniou ${ }^{3}$, Alison M. Dunning ${ }^{2}$, \\ Douglas F. Easton ${ }^{2,3}$ and Georgia Chenevix-Trench ${ }^{1}$ \\ 1 Department of Genetics and Computational Biology, QIMR Berghofer Medical Research Institute, \\ Brisbane QLD 4006, Australia; Wei.Shi@qimrberghofer.edu.au (W.S.); \\ Jonathan.Beesley@qimrberghofer.edu.au (J.B.); jaynelouise.pritchard@gmail.com (J.-L.P.); \\ Karen.McCue@qimrberghofer.edu.au (K.M.); Georgia.Trench@qimrberghofer.edu.au (G.C.-T.) \\ 2 Centre for Cancer Genetic Epidemiology, Department of Oncology, University of Cambridge, \\ Cambridge CB1 8RN, UK; lf9@sanger.ac.uk (L.F.); amd24@medschl.cam.ac.uk (A.M.D.); \\ dfe20@medschl.cam.ac.uk (D.F.E.) \\ 3 Centre for Cancer Genetic Epidemiology, Department of Public Health and Primary Care, \\ University of Cambridge, Cambridge CB1 8RN, UK; drb54@medschl.cam.ac.uk (D.R.B.); \\ aca20@medschl.cam.ac.uk (A.C.A.) \\ * Correspondence: dylan.glubb@qimr.edu.au; Tel.: +61-7-3845-3725
}

Received: 22 November 2019; Accepted: 30 December 2019; Published: 10 January 2020 updates

\begin{abstract}
Genome-wide association studies have revealed a locus at $8 \mathrm{p} 12$ that is associated with breast cancer risk. Fine-mapping of this locus identified 16 candidate causal variants (CCVs). However, as these variants are intergenic, their function is unclear. To map chromatin looping from this risk locus to a previously identified candidate target gene, DUSP4, we performed chromatin conformation capture analyses in normal and tumoural breast cell lines. We identified putative regulatory elements, containing CCVs, which looped to the DUSP4 promoter region. Using reporter gene assays, we found that the risk allele of CCV rs7461885 reduced the activity of a DUSP4 enhancer element, consistent with the function of DUSP4 as a tumour suppressor gene. Furthermore, the risk allele of CCV rs12155535, located in another DUSP4 enhancer element, was negatively correlated with looping of this element to the DUSP4 promoter region, suggesting that this allele would be associated with reduced expression. These findings provide the first evidence that $\mathrm{CCV}$ risk alleles downregulate DUSP4 expression, suggesting that this gene is a regulatory target of the 8p12 breast cancer risk locus.
\end{abstract}

Keywords: breast cancer risk; GWAS; candidate causal variant; chromatin conformation capture; reporter gene activity; enhancer; promoter

\section{Introduction}

Genome-wide association studies by the Breast Cancer Association Consortium (BCAC) and Consortium of Investigators of Modifiers of BRCA1/2 (CIMBA) have previously found genetic variants at 8 p12 associated with breast cancer risk [1-4]. In the largest study, the most significant association was for the rarer allele (A) of single nucleotide polymorphism rs9693444 with a per-allele odds ratio $(\mathrm{OR})=1.06\left(95 \% \mathrm{CI} 1.04-1.08 ; p=2 \times 10^{-21}\right)$ [1]. Subsequent fine-mapping analysis of BCAC and CIMBA data at this locus revealed 16 candidate causal variants (CCVs; Supplementary Table S1), including rs9693444, which could not be statistically separated from each other using a likelihood ratio criterion of 100:1 [5]. The CCVs are located in an intergenic region $\geq 300 \mathrm{~kb}$ centromerically from DUSP4 and $\geq 285 \mathrm{~kb}$ telomerically from the microRNA MIR3148. 
A major obstacle in the follow-up of genome-wide association study (GWAS) loci is the identification of target genes. Indeed, given the intergenic location of the CCVs at $8 \mathrm{p} 12$, it is unclear what function the risk variation may have. To identify breast cancer GWAS target genes, we previously developed a pipeline called INQUISIT (integrated expression quantitative trait and in silico prediction of GWAS targets). INQUISIT incorporates functional genomic data from breast cell lines and tissues, providing a score to prioritise identified target genes [3,5]. In our latest fine-mapping study, INQUISIT revealed seven target genes at the 8p12 breast cancer risk locus, of which only DUSP4 was a high confidence target [5]. This prediction was partly based on evidence of long-range regulatory chromatin interactions between CCVs and the DUSP4, promoter which included RNA polymerase II chromatin interaction analysis by paired-end tag sequencing (ChIA-PET) data in a breast cancer cell line [6], Hi-C chromatin-interaction and bioinformatic analysis identifying an enhancer coincident with CCVs that interacts with the DUSP4 promoter [7], and capture Hi-C data from breast cancer cell lines [8].

The chromatin interaction data identified through INQUISIT suggest that CCVs could regulate DUSP4 expression through enhancer-promoter interactions. This may be a common mechanism through which GWAS variants act (reviewed in [9]). Indeed, at a number of breast cancer GWAS risk loci, we have previously shown that CCVs in regulatory elements loop to promoters and regulate their activity $[3,10,11]$. In the current study, we mapped long-range chromatin interactions between the $8 \mathrm{p} 12$ risk locus and the promoter region of DUSP4. We provide evidence that a CCV, coincident with a putative regulatory element (PRE) that interacts with DUSP4, can regulate DUSP4 promoter activity. Furthermore, we found a CCV in a different PRE that was associated with allele-specific chromatin looping to the DUSP4 promoter region. These data suggest strongly that DUSP4 is a target gene of breast cancer risk variation at the $8 \mathrm{p} 12$ locus.

\section{Results}

2.1. Chromatin Conformation Capture (3C) Analyses Confirmed That the $8 p 12$ Risk Locus Physically Interacted with the Promoter Region of DUSP4

To map chromatin interactions between specific regions of the 8p12 risk locus (defined by EcoRI restriction fragments; Figure 1) and the DUSP4 promoter in breast cells, chromatin conformation capture (3C) analyses were conducted in breast cancer (MCF-7 and T-47D) and normal mammary epithelial (Bre-80) cell lines. Some level of interaction with the DUSP4 promoter was observed in all three cell lines across the $3 \mathrm{C}$ restriction fragments at the risk locus (Figure 1). Notably, this broad level of activity is not often observed in $3 \mathrm{C}$ analyses. For example, the $2 \mathrm{q} 35$ and $5 \mathrm{q} 11.2$ breast cancer risk loci are considerably closer $(\leq 100 \mathrm{~kb})$ to corresponding promoter targets than $8 \mathrm{p} 12 \mathrm{CCVs}$ are to the DUSP4 promoter region, yet many restriction fragments at these loci have no observable promoter interaction [10,11]. At the 8p12 locus, three 3C restriction fragments (\#10, \#11 and \#12) with Hi-C and/or ChIA-PET evidence of chromatin looping to the DUSP4 promoter demonstrated peaks of interaction in the breast cell lines (Figure 1), highlighting two fragments (\#11 and \#12) which contain CCVs (two and three, respectively) for further analysis. 


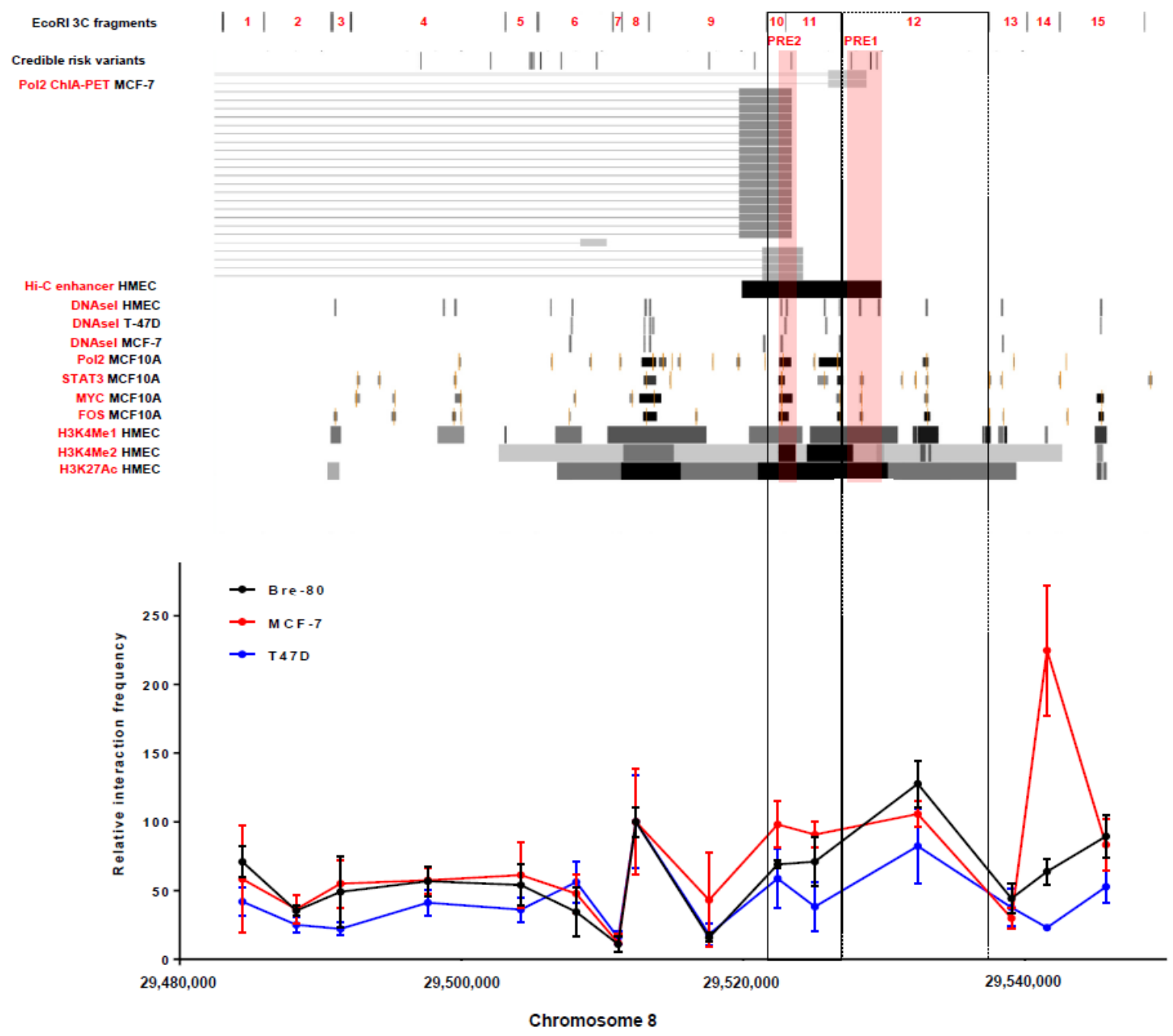

Figure 1. Candidate risk variants (CCVs) were located in putative regulatory elements (PREs) that interact with the DUSP4 promoter. The figure shows the 8p12 breast cancer risk locus annotated with breast cell functional genomic data and $3 \mathrm{C}$ analyses of interactions between EcoRI fragments and the DUSP4 promoter region (located $\geq 300 \mathrm{~kb}$ upstream) in Bre-80, MCF-7, and T-47D cells. Functional genomic data from normal mammary epithelial cells (HMEC and MCF-10A) and breast cancer cell lines (MCF-7 and T-47D) in this figure include Hi-C analysis [7] and ENCODE data accessed from the Univeristy of California Santa Cruz Genome Browser: RNA polymerase II chromatin interaction analysis by paired-end tag sequencing (ChIA-PET), epigenetic marks characteristic of enhancers (DNaseI hypersensitivity sites and H3K4Me1, H3K4Me2, and H3K27Ac histone modifications), and transcription factor binding (Pol2, STAT3, MYC, and FOS). Notably, all ChIA-PET anchors and the predicted Hi-C enhancer looped to the DUSP4 promoter. For the $3 \mathrm{C}$ analyses, interaction frequencies were normalised to those of fragment 8 , a common peak of interaction in all three cell lines. Interaction frequencies from three independent biological replicates are shown (error bars represent standard error of the mean). Fragments demonstrating interaction peaks and encompassing PREs with CCVs are bounded by boxes. The PRE regions cloned for reporter gene analysis are highlighted in pink.

\subsection{CCV rs7461885 Reduced the Enhancer Activity of PRE1 on the DUSP4 Promoter}

From analysis of the 3C, ChIA-PET, and epigenetic data at the 8p12 locus (Figure 1), CCVs in two putative regulatory elements (PRE1-2) were prioritized for reporter gene analyses to determine their effects on DUSP4 promoter activity (Figure 2). PRE1, containing three CCVs, significantly enhanced DUSP4 promoter activity by 2.3- and 2.5-fold in Bre-80 and MCF-7 cells, respectively (Figure 2a,b). In these two cell lines, the risk-associated allele of rs7461885, whether as a single variant or in a 
haplotype with the risk alleles of two other CCVs, significantly reduced the PRE1 enhancer activity by 1.3-1.5 fold (Figure 2a,b). Analysis of rs7461885 using HaploReg [12] did not indicate any evidence for transcription factor motifs or binding; however, this variant is located in active histone marks (H3K4Me1, H3K4Me2, and H3K27Ac) in normal mammary epithelial cells (Figure 1). PRE2, containing two CCVs, significantly enhanced DUSP4 promoter activity by 2.5- and 2.7-fold in MCF-7 and Bre-80 cells, respectively, but neither CCV affected this enhancer activity, nor did the haplotype containing both risk alleles (Figure 2c,d). Reporter assays were also performed in T-47D cells, but neither PREs nor CCVs significantly affected DUSP4 promoter activity (Supplementary Figure S1).
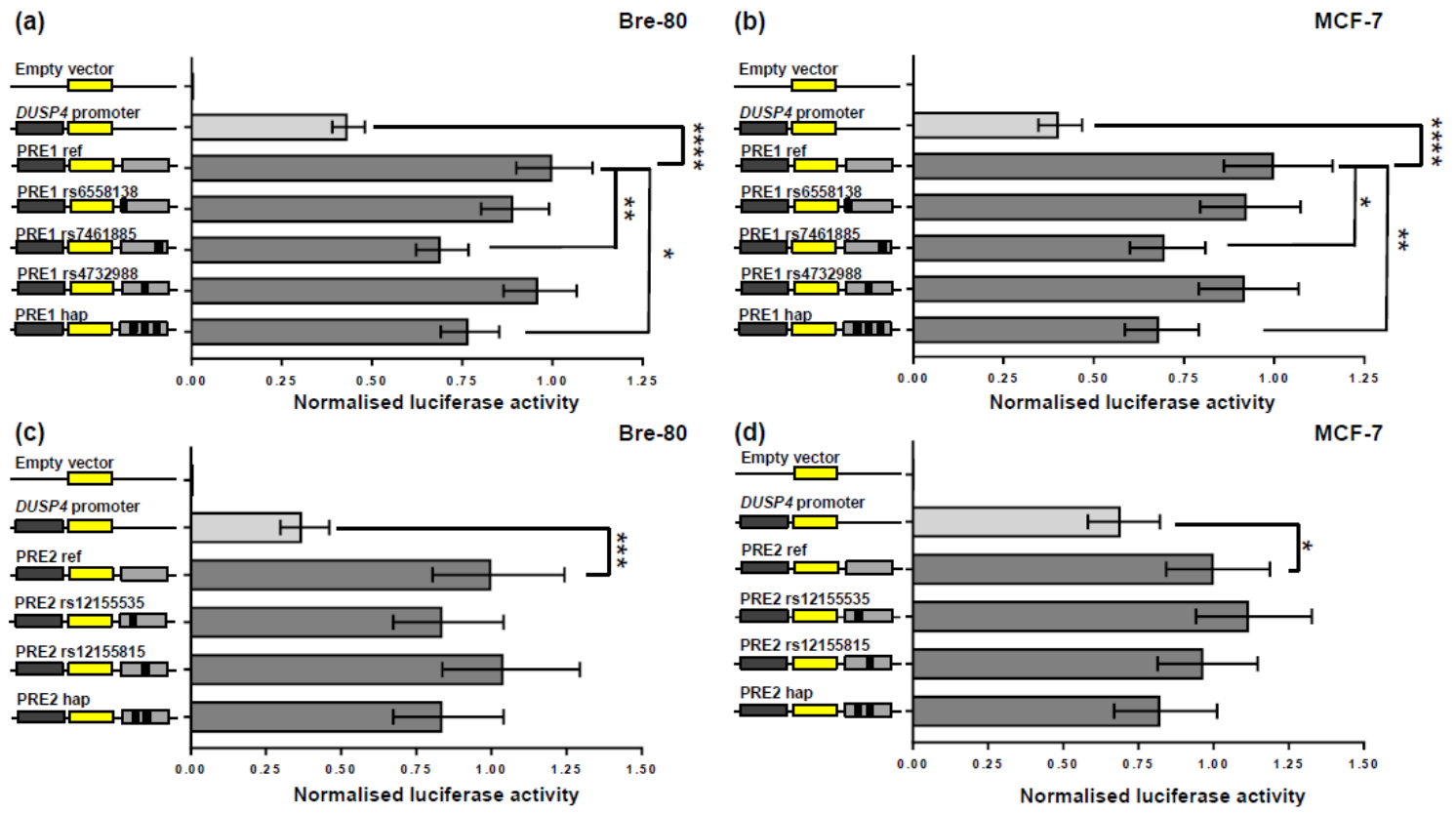

Figure 2. PREs enhanced DUSP4 promoter activity in luciferase reporter assays and the risk allele of CCV rs7461885 reduced putative regulatory element 1 (PRE1) enhancer activity. PRE1 and PRE2 reference regions, containing protective allelic variants of CCVs, were cloned downstream of a DUSP4 promoter luciferase construct for the creation of reference (ref) constructs. Risk allelic variants of $\mathrm{CCVs}$ were engineered into the constructs and were designated by the rs ID of the corresponding variant. Constructs containing risk allele haplotypes (hap) were also generated. Cells were transiently transfected with each of these constructs and assayed for luciferase activity after $24 \mathrm{~h}$. Panels show back-transformed data for (a) PRE1 activity in Bre-80 cells, (b) PRE1 activity in MCF-7 cells, (c) PRE2 activity in Bre- 80 cells, and (d) PRE2 activity in MCF-7 cells. Error bars denote $95 \%$ confidence intervals of experiments performed in triplicate. $p$-values were determined two-way ANOVA followed by Dunnett's multiple comparisons test $\left({ }^{*} p<0.05,{ }^{* *} p<0.01,{ }^{* * *} p<0.001\right.$ and $\left.{ }^{* * *} p<0.0001\right)$.

\subsection{CCV rs12155535 (PRE2) Was Associated with Allele-Specific Looping to the DUSP4 Promoter Region}

Of the three breast cell lines used in the 3C assay, only one (Bre-80) was heterozygous for CCVs and thus amenable for allele-specific $3 \mathrm{C}$ analysis. After assessing CCVs in the PREs and their proximity to the $3 C$ restriction sites, we found that only PRE2 could be analysed for allele-specific looping using PCR-based Sanger sequencing. Using Bre-80 3C libraries, we found that there was a reduction of the risk (C) allele of CCV rs12155535 in PRE2 interactions, compared to the Bre-80 genomic DNA control (Figure 3). This finding indicates that the risk allele was negatively correlated with the PRE2 enhancer-DUSP4 promoter region interaction, suggesting that risk allele may be associated with diminished DUSP4 expression through a reduction in enhancer looping to the DUSP4 promoter region. 


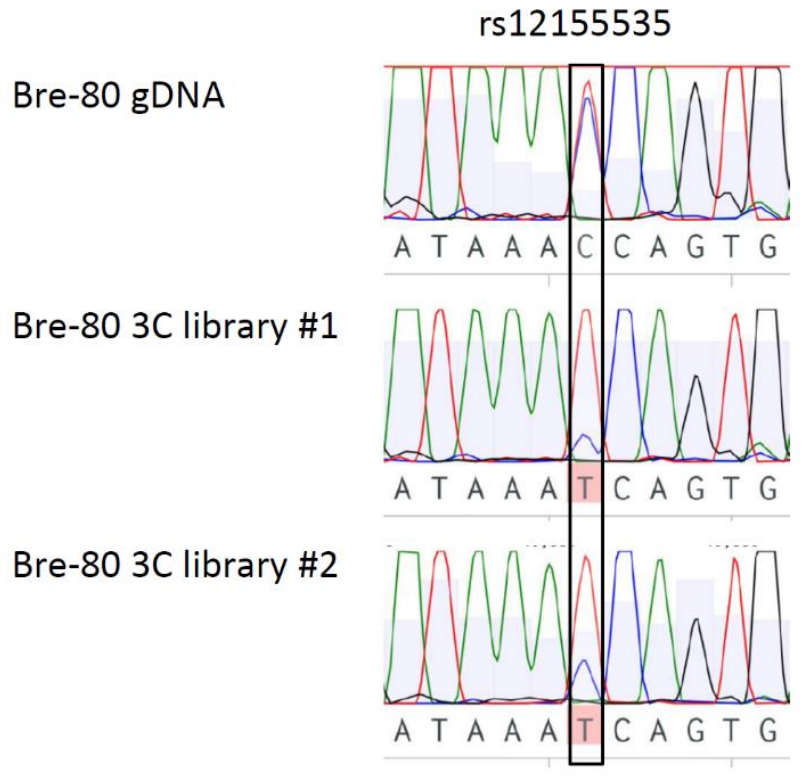

Figure 3. Allele-specific interaction between PRE2 and the DUSP4 promoter region. Bre-80 cells are heterozygous for the rs12155535 CCV in PRE2. Allele-specific analysis using PCR-based Sanger sequencing of two independent $3 \mathrm{C}$ libraries indicated that sequence containing the protective $(\mathrm{T})$ allele of rs12155535 preferentially interacted with the DUSP4 promoter region.

\section{Discussion}

In this study, we used genetic approaches to identify functional CCVs and regulatory targets at the 8 p12 breast cancer risk locus. Firstly, we performed $3 \mathrm{C}$ in normal and breast cancer cell lines to map chromatin interactions between PREs, containing CCVs, and the DUSP4 promoter region. Although we cannot rule out interactions with other genes at this locus, these findings were consistent with available chromatin interaction analyses, and no other high-confidence targets were predicted by the INQUISIT pipeline in this region [5].

Secondly, we prioritised two PREs with evidence of interaction with the DUSP4 promoter region (i.e., PRE1-2) for reporter gene analyses. These assays demonstrated that PRE1 and PRE2 acted as enhancers of DUSP4 promoter activity in both normal and tumoural breast cell lines. PRE1 and PRE2 are located in areas of active chromatin, as indicated by the presence of transcription factor binding and strong histone peaks characteristic of enhancers (Figure 1). CCVs in PRE1 and PRE2 were tested for effects on the DUSP4 promoter, and the risk allele of CCV rs7461885 was found to repress the DUSP4 enhancer activity of PRE1. It is possible that other CCVs at the 8p12 locus, which were not assessed in the reporter gene assays, may also regulate DUSP4; however, the available epigenetic data suggested these variants were less likely to be functional.

Finally, we performed an allele-specific $3 \mathrm{C}$ analysis and found that the risk allele of CCV rs12155535 was present less often in PRE2-DUSP4 promoter region interactions in comparison with the protective allele. As the reporter gene assays had demonstrated that PRE2 acted as a DUSP4 enhancer, the association of the rs12155535 risk allele with reduced PRE2-DUSP4 interaction frequency was consistent with the negative effect of the risk allele of rs7461885 on DUSP4 promoter activity.

The $3 \mathrm{C}$ and reporter gene findings indicate that DUSP4 is a likely target gene and is downregulated by risk associated variation at the $8 \mathrm{p} 12$ locus. A limitation of the reporter gene analysis is that because it is plasmid-based it does not model long-range interaction regulatory effects that occur in the human genome. Nonetheless, enhancers are understood to maintain their functions in reporter plasmids, despite the artificial genetic environment, and reporter assays are considered the "gold standard" for assessing enhancer activity [13]. Indeed, data suggest that distance is not essential for regulatory interaction in a plasmid-based assay-Tewhey et al. estimated a positive predictive value of up to $68 \%$ 
when validating expression quantitative trait loci (eQTLs) using reporter plasmids that incorporate only $150 \mathrm{bp}$ of enhancer sequence and a minimal promoter [14].

Expression quantitative trait loci data from mammary tissue $(n=396)$ in the Genotype Tissue Expression database (version 8; https://gtexportal.org/home/ accessed on 9 October 2019) and tumour tissue $(n=799)$ in The Cancer Genome Atlas eQTL browser (https://albertlab.shinyapps.io/tcga_eqt1/ accessed on 9 October 2019) provided no evidence that CCVs are associated with DUSP4 expression in normal or tumour breast tissue. A lack of CCV eQTLs at cancer risk GWAS loci is a common finding $[10,11,15,16]$ and could be due to several issues: (i) the available eQTL studies may not have enough statistical power to detect modest effects (as observed in the reporter gene assay) on DUSP4 expression, for example, eQTLs were found at a third of breast cancer GWAS risk loci only after combining data from four relevant eQTL studies ( $n=2820$ ) [17]; (ii) the tissues used, especially tumour tissue, were heterogeneous, containing multiple cell types, and thus may have masked CCV effects on DUSP4 expression that occur in specific cell types; and (iii) CCVs could have development or context-specific effects on DUSP4 expression that were not captured by the available studies.

DUSP4 encodes a nuclear dual specificity protein phosphatase that inactivates ERK, JNK, and p38 mitogen-activated protein kinases [18] and may also play a regulatory role in gene expression through chromatin binding [19]. DUSP4 is frequently deleted in breast tumours [20,21] and cancer cell lines [22]. DUSP4 knockdown enhances the formation of breast cancer stem cells $[19,23]$ and increases the invasive ability of estrogen receptor-positive breast cancer stem cells [23]. In ER-negative breast cancer cells, DUSP4 knockdown increases the formation of mammospheres and the expression of cancer stem cell promoting cytokines [22]. Thus, the literature indicates that DUSP4 protein acts as a tumour suppressor in breast cancer, which is compatible with our finding of its negative regulation by a breast cancer risk allele.

\section{Materials and Methods}

\subsection{Cell Culture}

MCF-7 and T-47D cell lines were purchased from ATCC (\#HTB22 and \#HTB-133, respectively), and Bre-80 was kindly provided by Roger Reddel (CMRI, Sydney, Australia). Cell lines were stored in liquid nitrogen vapour phase with Mycoplasma testing and short tandem repeat profiling performed for cell authentication prior to storage. The immortalised mammary epithelial Bre- 80 cell line was cultured as previously described [24]. The breast cancer cell lines MCF-7 and T-47D were cultured in RPMI 1640 medium supplemented with fetal calf serum, penicillin/streptomycin, and $10 \mu \mathrm{g} / \mathrm{ml}$ insulin. All cell lines were cultured at $37^{\circ} \mathrm{C}$ in a humidified $5 \% \mathrm{CO}_{2}$ atmosphere.

\subsection{Analysis}

$3 \mathrm{C}$ libraries were generated from MCF-7, T-47D, and Bre-80 cells. Briefly, cells were grown in $100 \mathrm{~mm}$ plates and fixed with $1 \%$ formaldehyde after reaching $\approx 80 \%$ confluency. Formaldehyde was quenched with ice-cold $0.125 \mathrm{M}$ glycine in phosphate buffered saline and cells collected by scraping and centrifugation. After washing with PBS, cells were incubated in ice-cold cell lysis buffer $(10 \mathrm{mM}$ Tris pH 7.5, $10 \mathrm{mM} \mathrm{NaCl}, 0.2 \%$ IGEPAL and cOmplete protease inhibitors (Roche)) for $30 \mathrm{~min}$ on ice, followed by 10 strokes of a Dounce homogenizer. Cell nuclei were collected by centrifugation and incubated overnight at $37^{\circ} \mathrm{C}$ with $1500 \mathrm{U}$ of EcoRI in New England Biolabs restriction buffer with $0.3 \%$ sodium dodecyl sulfate and $2 \%$ Triton-X 100. Restriction enzyme was heat-inactivated at $65^{\circ} \mathrm{C}$ for $20 \mathrm{~min}$ and ligation was performed in $8 \mathrm{~mL}$ of ligation buffer (1\% Triton X-100, 1.15× NEB ligation buffer, $0.1 \mathrm{mg} / \mathrm{mL}$ bovine serum albumin and $1 \mathrm{mM}$ ATP). $3 \mathrm{C}$ libraries were then phenol/chloroform extracted and precipitated with ethanol.

$3 \mathrm{C}$ interactions were quantified by qPCR with primers designed with the EcoRI restriction fragments spanning the 8p12 risk locus (Supplementary Table S2). qPCR was performed using a RotorGene 6000 with a reaction mix containing MyTaq HS DNA polymerase and the addition of $5 \mathrm{mM}$ 
Syto9. Thermal cycling was performed with an annealing temperature of $66^{\circ} \mathrm{C}(20 \mathrm{~s})$ and extension at $72{ }^{\circ} \mathrm{C}(30 \mathrm{~s})$. Three independent $3 \mathrm{C}$ libraries were analysed by qPCR with each experiment quantified in duplicate. Two bacterial artificial clones (RP11-56L5 and RP11-833O14: BACPAC Resource Center) encompassing the DUSP4 promoter region and the $8 \mathrm{p} 12$ breast cancer risk locus were used to create a library of ligation products to determine $3 \mathrm{C}$ primer efficiencies for normalization.

\subsection{Reporter Gene Vector Construction}

A DUSP4 promoter luciferase reporter construct was generated by inserting $1650 \mathrm{bp}$ of PCR-amplified DNA, containing the DUSP4 transcription start site (chr8:29,207,878-29,209,527; GRCh37), into the KpnI and HindIII sites of a pGL3-Basic construct. The pGL3-Basic construct had been engineered to include AgeI and SbfI sites downstream of the luciferase gene. A 2464 bp PRE1 region (chr88:29,527,323-29,530,033) was PCR generated using primers engineered with BamHI and SalI sites for insertion downstream of the Firefly luciferase gene. A 980 bp PRE2 region (chr8:29522728-29523707) was synthesised with terminal AgeI/SalI sites (Integrated DNA Technologies, Singapore, Singapore) for cloning into the DUSP4 promoter construct. For PRE1, the allelic variants of CCVs were introduced by overlap extension PCR. For PRE2, constructs containing allelic variants were also synthesised by Integrated DNA Technologies and cloned as above. All constructs were Sanger sequenced (QIMR Berghofer sequencing facility) to confirm variant incorporation. All PCR and sequencing primers are listed in Supplementary Table S3.

\subsection{Reporter Gene Analysis}

Bre-80, MCF-7, and T-47D cells were transfected with equimolar amounts of luciferase reporter constructs and $50 \mathrm{ng}$ of the Renilla luciferase pRL-SV40 construct with Lipofectamine 2000. The total amount of transfected DNA was kept constant at $600 \mathrm{ng}$ for each construct by adding pUC19 as a carrier plasmid. Luciferase activity was measured $24 \mathrm{~h}$ post-transfection by the Dual-Glo Luciferase Assay System. To correct for variation in transfection efficiency or cell lysate preparation, Firefly luciferase activity was normalised to that of the Renilla luciferase. All DUSP4 promoter constructs had great activity compared with the negative control (empty pGL3-Basic construct). Data were log-transformed and statistical significance was assessed by two-way ANOVA, followed by Dunnett's multiple comparisons test in GraphPad Prism (version 7.02, GraphPad Software, San Diego, CA, USA).

\subsection{Allele-Specific 3C Analysis}

DNA was amplified by PCR of two independent Bre-80 3C libraries using the DUSP4 promoter bait primer and a reverse primer specific to the PRE2 fragment containing rs12155535 (Supplementary Table S2). The region containing rs12155535 was also amplified from Bre-80 genomic DNA as a control. Sanger sequencing (QIMR Berghofer sequencing facility) was performed to determine the alleles present in the interacting PRE2 fragment.

\section{Conclusions}

We found the first evidence that breast cancer risk variation at the 8p12 locus downregulates DUSP4 promoter activity and is negatively correlated with long-range chromatin looping interactions with the DUSP4 promoter region. These findings are consistent with the role of DUSP4 as a tumour suppressor in breast cancer and suggest that the effects of CCVs on breast cancer risk may be mediated by a reduction in DUSP4 expression.

Supplementary Materials: The following are available online at http://www.mdpi.com/2072-6694/12/1/170/s1. Figure S1: Luciferase reporter gene analysis of PRE1-3 in T-47D cells, Table S1: 8p12 CCVs, Table S2: 3C primers, Table S3: Primers for generation of reporter gene constructs.

Author Contributions: Conceptualization, D.M.G. and G.C.-T.; formal analysis, W.S., J.B. and D.M.G.; investigation, W.S., J.-L.P., K.M., J.B. and D.M.G.; resources, D.F.E. and G.C.-T.; data curation, D.M.G., L.F. and J.B.; writing—original draft preparation, D.M.G. and G.C.-T.; writing—review and editing, D.M.G., W.S., J.B., 
L.F., J.-L.P., K.M., D.R.B., A.C.A., A.M.D., D.F.E. and G.C.-T.; visualization, D.M.G.; supervision, D.M.G., K.M., A.C.A., A.M.D., D.F.E. and G.C.-T.; project administration, G.C.-T.; funding acquisition, G.C.-T. All authors have read and agreed to the published version of the manuscript.

Funding: This research was funded by the National Health and Medical Research Council of Australia.

Acknowledgments: The authors acknowledge the BCAC and CIMBA consortia in providing pre-publication access to the fine-mapping data.

Conflicts of Interest: The authors declare no conflict of interest. The funders had no role in the design of the study; in the collection, analyses, or interpretation of data; in the writing of the manuscript, or in the decision to publish the results.

\section{References}

1. Michailidou, K.; Beesley, J.; Lindstrom, S.; Canisius, S.; Dennis, J.; Lush, M.J.; Maranian, M.J.; Bolla, M.K.; Wang, Q.; Shah, M.; et al. Genome-wide association analysis of more than 120,000 individuals identifies 15 new susceptibility loci for breast cancer. Nat. Genet. 2015, 47, 373-380. [CrossRef]

2. Michailidou, K.; Hall, P.; Gonzalez-Neira, A.; Ghoussaini, M.; Dennis, J.; Milne, R.L. Large-scale genotyping identifies $41 \mathrm{new}$ loci associated with breast cancer risk. Nat. Genet. 2013, 45. [CrossRef]

3. Michailidou, K.; Lindstrom, S.; Dennis, J.; Beesley, J.; Hui, S.; Kar, S.; Lemacon, A.; Soucy, P.; Glubb, D.; Rostamianfar, A.; et al. Association analysis identifies 65 new breast cancer risk loci. Nature 2017, 551, 92-94. [CrossRef]

4. Mulligan, A.M.; Couch, F.J.; Barrowdale, D.; Domchek, S.M.; Eccles, D.; Nevanlinna, H.; Ramus, S.J.; Robson, M.; Sherman, M.; Spurdle, A.B.; et al. Common breast cancer susceptibility alleles are associated with tumour subtypes in BRCA1 and BRCA2 mutation carriers: Results from the Consortium of Investigators of Modifiers of BRCA1/2. Breast Cancer Res. 2011, 13, R110. [CrossRef]

5. Fachal, L.; Aschard, H.; Beesley, J.; Barnes, D.R.; Allen, J.; Kar, S.; Pooley, K.A.; Dennis, J.; Michailidou, K.; Turman, C.; et al. Fine-mapping of 150 breast cancer risk regions identifies 178 high confidence target genes. bioRxiv 2019, 521054. [CrossRef]

6. Li, G.; Ruan, X.; Auerbach, R.K.; Sandhu, K.S.; Zheng, M.; Wang, P.; Poh, H.M.; Goh, Y.; Lim, J.; Zhang, J.; et al. Extensive promoter-centered chromatin interactions provide a topological basis for transcription regulation. Cell 2012, 148, 84-98. [CrossRef] [PubMed]

7. Rao, S.S.; Huntley, M.H.; Durand, N.C.; Stamenova, E.K.; Bochkov, I.D.; Robinson, J.T.; Sanborn, A.L.; Machol, I.; Omer, A.D.; Lander, E.S.; et al. A 3D map of the human genome at kilobase resolution reveals principles of chromatin looping. Cell 2014, 159, 1665-1680. [CrossRef] [PubMed]

8. Beesley, J.; Sivakumaran, H.; Marjaneh, M.M.; Lima, L.G.; Hillman, K.M.; Kaufmann, S.; Tuano, N.; Hussein, N.; Ham, S.; Mukhopadhyay, P.; et al. Chromatin interactome mapping at 139 independent breast cancer risk signals. bioRxiv 2019, 520916. [CrossRef] [PubMed]

9. Gallagher, M.D.; Chen-Plotkin, A.S. The Post-GWAS Era: From Association to Function. Am. J. Hum. Genet. 2018, 102, 717-730. [CrossRef] [PubMed]

10. Glubb, D.M.; Maranian, M.J.; Michailidou, K.; Pooley, K.A.; Meyer, K.B.; Kar, S.; Carlebur, S.; O’Reilly, M.; Betts, J.A.; Hillman, K.M.; et al. Fine-Scale Mapping of the 5q11.2 Breast Cancer Locus Reveals at Least Three Independent Risk Variants Regulating MAP3K1. Am. J. Hum. Genet. 2015, 96, 5-20. [CrossRef]

11. Ghoussaini, M.; Edwards, S.L.; Michailidou, K.; Nord, S.; Cowper-Sal Lari, R.; Desai, K.; Kar, S.; Hillman, K.M.; Kaufmann, S.; Glubb, D.M.; et al. Evidence that breast cancer risk at the 2q35 locus is mediated through IGFBP5 regulation. Nat. Commun. 2014, 4, 4999. [CrossRef]

12. Ward, L.D.; Kellis, M. HaploReg: A resource for exploring chromatin states, conservation, and regulatory motif alterations within sets of genetically linked variants. Nucleic Acids Res. 2012, 40, D930-D934. [CrossRef] [PubMed]

13. Shlyueva, D.; Stampfel, G.; Stark, A. Transcriptional enhancers: From properties to genome-wide predictions. Nat. Rev. Genet. 2014, 15, 272-286. [CrossRef] [PubMed]

14. Tewhey, R.; Kotliar, D.; Park, D.S.; Liu, B.; Winnicki, S.; Reilly, S.K.; Andersen, K.G.; Mikkelsen, T.S.; Lander, E.S.; Schaffner, S.F.; et al. Direct Identification of Hundreds of Expression-Modulating Variants using a Multiplexed Reporter Assay. Cell 2016, 165, 1519-1529. [CrossRef] [PubMed] 
15. O'Mara, T.A.; Glubb, D.M.; Kho, P.F.; Thompson, D.J.; Spurdle, A. Genome-wide association studies of endometrial cancer: Latest developments and future directions. Cancer Epidemiol. Biomark. Prev. 2019, 28, 1095-1102. [CrossRef]

16. Lawrenson, K.; Li, Q.; Kar, S.; Seo, J.H.; Tyrer, J.; Spindler, T.J.; Lee, J.; Chen, Y.; Karst, A.; Drapkin, R.; et al. Cis-eQTL analysis and functional validation of candidate susceptibility genes for high-grade serous ovarian cancer. Nat. Commun. 2015, 6, 8234. [CrossRef]

17. Guo, X.; Lin, W.; Bao, J.; Cai, Q.; Pan, X.; Bai, M.; Yuan, Y.; Shi, J.; Sun, Y.; Han, M.-R.; et al. A Comprehensive cis-eQTL Analysis Revealed Target Genes in Breast Cancer Susceptibility Loci Identified in Genome-wide Association Studies. Am. J. Hum. Genet. 2018, 102, 890-903. [CrossRef]

18. Keyse, S.M. Dual-specificity MAP kinase phosphatases (MKPs) and cancer. Cancer Metastasis Rev. 2008, 27, 253-261. [CrossRef]

19. Boulding, T.; Wu, F.; McCuaig, R.; Dunn, J.; Sutton, C.R.; Hardy, K.; Tu, W.; Bullman, A.; Yip, D.; Dahlstrom, J.E.; et al. Differential Roles for DUSP Family Members in Epithelial-to-Mesenchymal Transition and Cancer Stem Cell Regulation in Breast Cancer. PLoS ONE 2016, 11, e0148065. [CrossRef]

20. Armes, J.E.; Hammet, F.; de Silva, M.; Ciciulla, J.; Ramus, S.J.; Soo, W.K.; Mahoney, A.; Yarovaya, N.; Henderson, M.A.; Gish, K.; et al. Candidate tumor-suppressor genes on chromosome arm 8p in early-onset and high-grade breast cancers. Oncogene 2004, 23, 5697-5702. [CrossRef]

21. Mazumdar, A.; Poage, G.M.; Shepherd, J.; Tsimelzon, A.; Hartman, Z.C.; Den Hollander, P.; Hill, J.; Zhang, Y.; Chang, J.; Hilsenbeck, S.G.; et al. Analysis of phosphatases in ER-negative breast cancers identifies DUSP4 as a critical regulator of growth and invasion. Breast Cancer Res. Treat. 2016, 158, 441-454. [CrossRef] [PubMed]

22. Balko, J.M.; Schwarz, L.J.; Bhola, N.E.; Kurupi, R.; Owens, P.; Miller, T.W.; Gomez, H.; Cook, R.S.; Arteaga, C.L. Activation of MAPK pathways due to DUSP4 loss promotes cancer stem cell-like phenotypes in basal-like breast cancer. Cancer Res. 2013, 73, 6346-6358. [CrossRef] [PubMed]

23. Gaggianesi, M.; Turdo, A.; Chinnici, A.; Lipari, E.; Apuzzo, T.; Benfante, A.; Sperduti, I.; Di Franco, S.; Meraviglia, S.; Lo Presti, E.; et al. IL4 Primes the Dynamics of Breast Cancer Progression via DUSP4 Inhibition. Cancer Res. 2017, 77, 3268-3279. [CrossRef] [PubMed]

24. Meyer, K.B.; O’Reilly, M.; Michailidou, K.; Carlebur, S.; Edwards, S.L.; French, J.D.; Prathalingham, R.; Dennis, J.; Bolla, M.K.; Wang, Q.; et al. Fine-Scale Mapping of the FGFR2 Breast Cancer Risk Locus: Putative Functional Variants Differentially Bind FOXA1 and E2F1. Am. J. Hum. Genet. 2013, 93, 1046-1060. [CrossRef] 